\title{
GEAMP, a novel gastroesophageal junction carcinoma cell line derived from a malignant pleural effusion
}

\author{
Wei Zhang ${ }^{1}$ - Taylor A. Williams ${ }^{1} \cdot$ Ankur S. Bhagwath $^{1} \cdot$ Jared S. Hiermann ${ }^{2} \cdot$ Craig D. Peacock $^{3}$ D. Neil Watkins ${ }^{4}$. \\ Peiguo Ding ${ }^{1} \cdot$ Jason Y. Park ${ }^{5} \cdot$ Elizabeth A. Montgomery $^{6} \cdot$ Arlene A. Forastiere $^{7} \cdot$ Chunfa Jie $^{8} \cdot$ Brandi L. Cantarel $^{9}$. \\ Thai H. Pham ${ }^{10,11} \cdot$ David H. Wang $\mathbb{1}^{1,11}$
}

Received: 14 March 2019 / Revised: 6 May 2019 / Accepted: 6 May 2019 / Published online: 10 July 2019

(c) The Author(s), under exclusive licence to United States and Canadian Academy of Pathology 2019

\begin{abstract}
Gastroesophageal junction (GEJ) cancer remains a clinically significant disease in Western countries due to its increasing incidence, which mirrors that of esophageal cancer, and poor prognosis. To develop novel and effective approaches for prevention, early detection, and treatment of patients with GEJ cancer, a better understanding of the mechanisms driving pathogenesis and malignant progression of this disease is required. These efforts have been limited by the small number of available cell lines and appropriate preclinical animal models for in vitro and in vivo studies. We have established and characterized a novel GEJ cancer cell line, GEAMP, derived from the malignant pleural effusion of a previously treated GEJ cancer patient. Comprehensive genetic analyses confirmed a clonal relationship between GEAMP cells and the primary tumor. Targeted next-generation sequencing identified 56 nonsynonymous alterations in 51 genes including TP53 and APC, which are commonly altered in GEJ cancer. In addition, multiple copy-number alterations were found including $E G F R$ and $K-R A S$ gene amplifications and loss of $C D K N 2 A$ and $C D K N 2 B$. Histological examination of subcutaneous flank xenografts in nude and NOD-SCID mice showed a carcinoma with mixed squamous and glandular differentiation, suggesting GEAMP cells contain a subpopulation with multipotent potential. Finally, pharmacologic inhibition of the EGFR signaling pathway led to downregulation of key downstream kinases and inhibition of cell proliferation in vitro. Thus, GEAMP represents a valuable addition to the limited number of bona fide GEJ cancer cell lines.
\end{abstract}

Supplementary information The online version of this article (https:// doi.org/10.1038/s41374-019-0278-x) contains supplementary material, which is available to authorized users.

David H. Wang

David1.Wang@UTSouthwestern.edu

1 Esophageal Diseases Center and Division of HematologyOncology, Department of Internal Medicine and the Simmons Comprehensive Cancer Center, University of Texas Southwestern Medical Center, Dallas, TX, USA

2 Department of Biomedical Engineering, University of Minnesota, Minneapolis, MN, USA

3 Translational Hematology and Oncology Research, Cleveland Clinic, Cleveland, OH, USA

4 The Kinghorn Cancer Centre, Garvan Institute of Medical Research, Darlinghurst, NSW, Australia

5 Department of Pathology and the Eugene McDermott Center for Human Growth and Development, UT Southwestern Medical Center, Dallas, TX, USA

\section{Introduction}

Esophageal cancer is the sixth leading cause of cancer death worldwide with the number of deaths estimated to be greater

6 Division of Gastrointestinal and Liver Pathology, Department of Pathology, Johns Hopkins Hospital, Baltimore, MD, USA

7 Department of Oncology, Johns Hopkins University School of Medicine, Baltimore, MD, USA

8 Department of Biochemistry and Nutrition, Des Moines University, Des Moines, IA, USA

9 Bioinformatics Core Facility, Lyda Hill Department of Bioinformatics, University of Texas Southwestern Medical Center, Dallas, TX, USA

10 Esophageal Diseases Center and Department of Surgery, University of Texas Southwestern Medical Center, Dallas, TX, USA

11 VA North Texas Health Care System, Dallas, TX, USA 
than 500,000 annually while gastric cancer is the third leading cause of cancer death worldwide with the number of deaths estimated to be greater than 700,000 annually $[1,2]$. There are two main histologic subtypes of esophageal cancer: squamous cell carcinoma, which is predominantly found in the upper and middle thirds of the esophagus, and adenocarcinoma, which typically arises in the lower esophagus and can involve the gastroesophageal junction (GEJ). In the past, tumors of the GEJ were classified as gastric cancers. However, the natural behavior and clinical and epidemiologic features of GEJ cancer are more similar to esophageal cancer [3]. As a result, in 2010, the Union for International Cancer Control moved GEJ cancers from the staging system of gastric cancer to the staging system for esophageal cancer [4]. Clinical treatment guidelines also now group GEJ cancer with esophageal cancer rather than gastric cancer [5]. The incidence of esophageal adenocarcinoma (EAC) and GEJ carcinoma has increased dramatically in the United States and other Western countries in the past three decades [6, 7]. While the exact causes for this are largely unknown, gastroesophageal reflux disease (GERD), Barrett's esophagus (BE), obesity, and tobacco use are the major risk factors [8-10]. In particular, BE, the metaplastic change of the squamous epithelium lining the distal esophagus into columnar epithelium that occurs as a complication of GERD, is a well-recognized premalignant lesion for EAC [11] but may be less so in GEJ cancer [12].

Despite recent advances in surgical technique, radiation therapy and chemotherapy regimens, long-term survival of EAC and GEJ tumors remains poor. The overall 5-year survival rate for all stages of EAC is below 20\% [13-15] while that of gastric cancer is $31 \%$ [15]. The recent rise in EAC and GEJ cancer incidence has resulted in renewed research efforts into the biology and genetics of these tumors. Recent studies using next-generation sequencing (NGS) have shown that EAC and GEJ cancers are some of the most highly mutated solid tumors and have a high degree of heterogeneity [16]. The recent TCGA study identified important mutations in TP53, CDKN2A, ARIDIA, $S M A D 4$, and $E R B B 2$ in EAC [16]. Notably, these findings are consistent with previous discoveries that mutations of CDKN2A and TP53 are present in dysplastic BE [17]. Mutations of ERBB2 in tumors lacking ERBB2 amplification can also occur, exemplifying an alternative mechanism of activation of the ERBB2-directed signaling pathway [16, 18]. In GEJ tumors, RUNX1, SMAD4, CDKN2A, FHIT, ERRB2, c-MYC, VEGFA, APC, and TP53 are among the most frequently mutated genes [16, 19].

To date, the exact cellular origins of BE, EAC, and GEJ cancer remain controversial [8, 11]. Previously, Quante et al. [20] established an inflammation-dependent transgenic mouse model that develops metaplasia, dysplasia, and adenocarcinoma at the mouse squamocolumnar junction (SCJ) following overexpression of IL-1 $\beta$ in the squamous esophageal and forestomach mucosa. They proposed that progenitor cells from the gastric cardia could move proximally into the distal esophagus and give rise to early BE lesions and presumably EAC later. Other animal models and clinical studies using patient specimens support a cellular origin of $\mathrm{BE}$, and presumably of EAC too, as stem or progenitor cells located at the GEJ. A different mouse model characterized by Wang et al. [21] found that p63-deficient neonatal mice exhibited a BE-like columnar epithelium that stained positively with Alcian blue and periodic acid-Schiff at the SCJ [21]. More recently, Jiang et al. [22] demonstrated that overexpression of $\mathrm{Cdx} 2$ in a population of transitional basal progenitor cells at the mouse SCJ led to intestinalized metaplasia resembling BE. To date, no purely genetically engineered animal models develop EAC or GEJ cancer and only a few human EAC and GEJ cancer cell lines are available for in vitro preclinical studies. The lack of widely accepted EAC and GEJ cancer mouse models underscores the importance of developing human EAC and GEJ cancer cell lines; however, several human EAC and GEJ cancer cell lines frequently used for research (SEG-1, BIC-1, and SK-GT-5) were shown to be derived from other tumor types [23]. Use of cell lines originating from nonesophageal tissue complicates the effort to develop effective treatment strategies for EAC and GEJ cancer. Currently, the verified cell lines derived from human primary EAC or GEJ cancer include FLO-1 [24], KYAE-1 [25], SK-GT-4 [26], OE19 [27], OE33 [27], JHEsoAd1 [28], OACP4C [29], OACM5.1 [29], ESO26 [30], ESO51 [23], OANC1 [31], and MFD-1 [32]. All of these cell lines were derived from human primary tumors except for KYAE-1 and OACM5.1. KYAE-1 was established from a malignant pleural effusion whereas OACM5.1 was generated from a metastatic lymph node of an EAC patient, respectively [33].

We have successfully established (and validated) a novel, tumorigenic GEJ cancer cell line, GEAMP (Gastro Esophageal Adenocarcinoma derived from a Malignant Pleural effusion), from a patient with metastatic GEJ cancer. Short tandem repeat (STR) profiling-based genetic analysis confirmed a clonal relationship between the primary GEJ tumor and GEAMP cells. Targeted gene sequencing of cancer-related genes identified: (1) 56 nonsynonymous variants in $\sim 51$ genes including TP53 and $A P C$; and (2) multiple copy number alterations including amplification of EGFR and $K-R A S$ and loss of $C D K N 2 A$ and $C D K N 2 B$. Furthermore, we examined morphological characteristics and functional behavior in vitro and in vivo, and tested GEAMP cells response to EGFR pathway pharmacologic inhibitors. This novel cell line may be a valuable preclinical model for GEJ cancer, in particular metastatic GEJ cancer. 


\section{Materials and methods}

\section{Clinical specimen}

Patient tissue procurement was approved by the Institutional Review Board of the Johns Hopkins University, Baltimore, MD and mouse xenograft experiments were approved by the Institutional Animal Care and Use Committee of both the Johns Hopkins University and the VA North Texas Health Care System, Dallas, TX.

Therapeutic thoracentesis was performed on a GEJ cancer patient with a malignant pleural effusion and the fluid subjected to a cytospin. The cells were then aseptically mixed with matrigel and injected into the bilateral flanks of a NOD-SCID mouse. Subsequent xenografts were passaged into the flanks of nude mice with some of the xenograft tissue used to generate an early passage cell line (GEAMP).

\section{Cell culture}

Early passage cells were grown using HITES media with 5\% FBS. For inhibitor studies, cells were changed into Advanced RPMI media with $1 \%$ serum. After preculture for $24 \mathrm{~h}$, GEAMP cells were incubated with DMSO, erlotinib, or lapatinib at a final concentration of $5-10 \mu \mathrm{M}$ in six-well plate. Cells were then harvested up to $120 \mathrm{~h}$ and counted using Trypan blue.

\section{DNA fingerprinting}

The relationship between the original tumor and the GEAMP cell line was determined by comparing the STR profiles of the two respective samples.

Genomic DNA was isolated from the formalin-fixed paraffin embedded primary tumor and GEAMP cells using the QIAamp DNA FFPE Tissue Kit and Qiagen Blood \& Cell Culture DNA Mini Kit, respectively, according to the manufacturer's instructions. Extracted DNA samples were then analyzed using the PowerPlex 16 Assay (Promega) by the UT Southwestern McDermott Center Sequencing Core.

\section{Targeted sequencing and copy number variation analysis}

Targeted sequencing of GEAMP cells was performed by the Genomics and Molecular Pathology Core at UT Southwestern Medical Center. This UT Southwestern Pan-Cancer NGS assay covers the exons of 1385 cancer-related genes. BWA-MEM [34] was used to align sequence reads to reference genome GRCh38 with subsequent processing by Samtools [35] and Picard (https://broadinstitute.github.io/ picard) to ensure proper file formatting and mark duplicates. Alignments were then recalibrated and realigned using
GATK [36, 37]. To detect genome germline variants, GATK [36, 37], Platypus [38], Samtools [35], and Speedseq [39] were used in version 1 of the assay. In version 2, GATK [36, 37] was replaced by Streka2 [40]. To detect somatic variants, Mutect2 [41], Speedseq [39], Shimmer [42], Varscan [43], and Virmid [44] were used. The effect of SNPs and INDELs on genes was predicted using snpEff [45] using the GRCh38.82 database. Allele frequency in the general population was determined by the ExAC database. Gene fusions (translocations) were detected using STARFusion [46]. CNVs were detected using CNVKit [47] an internally derived panel of 20 nontumor FFPE and blood samples. To filter false positive calls, we employed the following filtering method consistent with other clinical laboratories [48]. Variants present in $>5$ samples in COSMIC were filtered out if there were (1) less than 3 supporting alternative reads; (2) less than 10 total reads; (3) minor allele frequency less than 0.01 . Variants present in $\leq 5 \mathrm{sam}$ ples in COSMIC were filtered out if there were (1) less than 8 supporting alternative reads; (2) less than 10 total reads; (3) minor allele frequency less than 0.05 . To further identify possible disease causing variants, we removed variants in noncoding regions, those in known repeat regions (UCSC Simple Repeat Table) and those in $>1 \%$ of the general population using GNOMAD [49].

\section{Quantitative reverse transcriptase-PCR}

Total RNA was isolated with Trizol (Invitrogen) and then reverse-transcribed using the Quantitect kit (Qiagen). Quantitative real-time PCR (qRT-PCR) was performed using SYBR-Green Supermix (Bio-Rad) on an Applied Biosystems StepOnePlus machine. Relative amounts of complementary DNA were calculated using the $\Delta \Delta \mathrm{Ct}$ method and normalized to GAPDH. The primer sequences are as follows: LGR5 (F:5'-TGCTGGCTGGTGTG GATGCG-3'; R:5'-CACCCAGCAGGGGAACTGCG-3'), CD133 (F:5'-TGGCCCTCGTACTCGGCTCC-3'; R:5'-CA GCAGCCCCAGGACACAGC-3'), BMI1 (F: 5'-TTGGTG GTTACCGCTGGGGC-3'; R:5'-GCCAACAGCCCAGCA GGAGG-3'), and GAPDH (F: 5'-TGGGCTACACTGAGC ACCAG-3'; R: 5'-GGGTGTCGCTGTTGAAGTCA-3').

\section{Western blot}

The cells were lysed in cell lysis buffer $(50 \mathrm{mM}$ Tris- $\mathrm{HCl}$, $\mathrm{pH} 8.0,150 \mathrm{mM} \mathrm{NaCl}, 0.5 \%$ sodium deoxycholate, $1 \%$ Triton X-100 plus protease and phosphatase inhibitors; Thermo Scientific). Overall $20 \mu \mathrm{g}$ of protein was run on 4-12\% Bis-Tris gels and transferred to PVDF membranes. After blocking, the following antibodies were used to detect proteins: anti-phospho-EGFR (Cell Signaling), anti-EGFR (Cell Signaling), anti-phospho-ERK (Cell Signaling), 
Fig. 1 Representative images of $\mathrm{H} \& \mathrm{E}$ and ERBB2

immunohistochemical staining and FISH in the primary GEJ cancer tissue. Low $(\mathbf{a}: \times 100)$ and high $(\mathbf{b}: \times 400)$ magnification images of H\&E stained section of the primary GEJ tumor. c Negative ERBB2

immunohistochemical staining in the primary GEJ tumor (×400). d Negative ERBB2 gene amplification by FISH. Tissue section showing ERBB2 gene copies (red) and chromosome enumeration probe 17 (CEP17) copies (green)

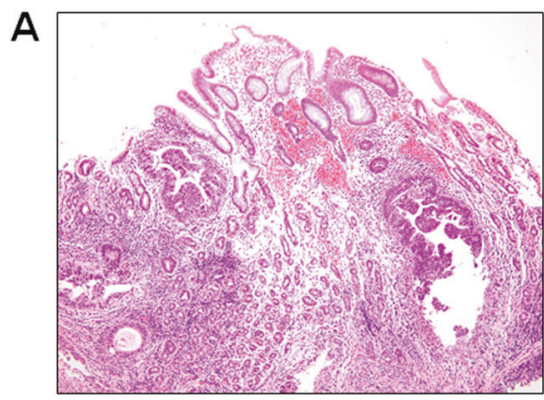

B

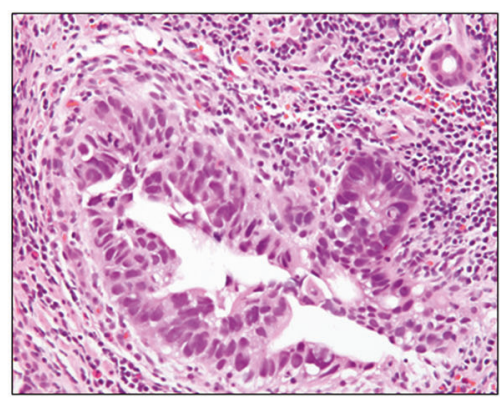

C

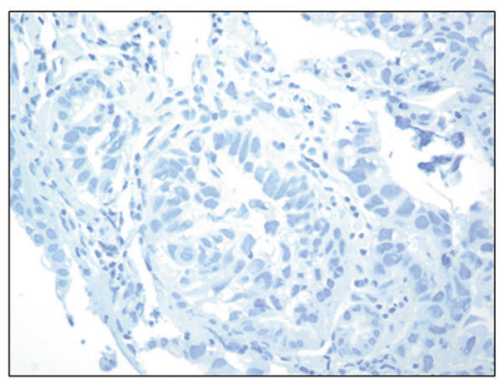

D

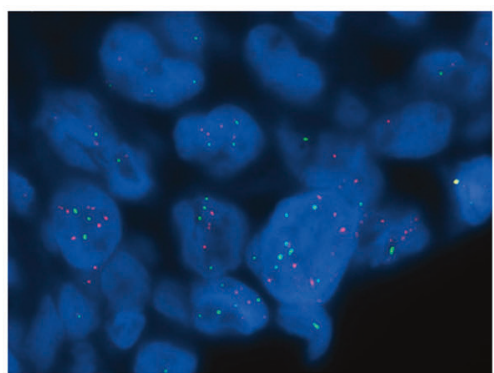

anti-ERK (Cell Signaling), anti-phospho-AKT (Santa Cruz Biotechnology), anti-AKT (Santa Cruz Biotechnology), anti-CK8/18 (Santa Cruz Biotechnology), anti-CK13 (Santa Cruz Biotechnology), anti-CK14 (BioLegend), and antiGAPDH (Millipore)

\section{Immunohistochemistry and fluorescent in situ hybridization}

Mouse tissue sections were stained with $\mathrm{H} \& \mathrm{E}$ or Alcian blue using standard procedures for morphological assessment. Immunohistochemistry was performed as previously described [50]. Antigen retrieval for deparaffinized tissue sections was performed in acidic citrate buffer for $20 \mathrm{~min}$ by using a steamer. Following the blocking of endogenous peroxidase, paraffin-embedded sections were submitted to immunohistochemistry using the Vectastain $\mathrm{ABC}$ system (Vector Laboratories, Burlingame, CA, USA) and visualized with DAB (Sigma). The following antibodies were used at the indicated dilutions: CK8/18 TROMA-I (Developmental Studies Hybridoma Bank, 1:100), CK14 (BioLegend 1:5000), CDX2 (BioGenex Clone CDX2-88 1:100), and TTF-1 (DAKO clone 8G7G3/1 1:1000). Immunostaining and FISH for ERRB2 and EGFR were performed by a clinical laboratory (ProPath; Dallas, TX). FISH was evaluated by enumerating the number of ERRB2 probe signals and CEP17 probe signals in 50 interphase nuclei to produce an average ERRB2:CEP17 ratio for the sample.

\section{Tumor xenografts}

To establish flank xenografts of GEAMP cells, $5 \times 10^{6}$ cells suspended in $100 \mu \mathrm{l}$ of PBS were mixed with $100 \mu \mathrm{l}$ matrigel (Corning), and subcutaneously injected into the flanks of female nude or NOD-SCID mice. Tumor volume was determined using the formula (length $\times$ width $\times$ width)/ 2 . The mice were euthanized when the tumors reached $\geq 1500 \mathrm{~mm}^{3}$.

\section{Results}

\section{Patient source}

The cell line was established from a 46-year-old white male who had been diagnosed with a T2N0M0 (stage IIA, AJCC 8th ed.) distal esophageal/GEJ adenocarcinoma (Fig. 1a, b). At that time, he presented with a 2-month history of progressive solid food dysphagia and a $5 \mathrm{lb}$ weight loss. He was otherwise in good health, took no medications, and denied symptoms of chronic reflux or antacid use. He was a nonsmoker and used alcohol only socially on weekends. He exercised regularly, running $\sim 3$ miles daily. Endoscopy revealed normal upper and middle esophageal mucosa. The GE junction was located $40 \mathrm{~cm}$ from the incisors. An exophytic tumor with a broad base extended from 40 to $43 \mathrm{~cm}$ from the incisors. The mass occupied less than one-fourth of the circumference of the cardia. The depth of invasion by endoscopic ultrasound involved the mucosa, submucosa, and muscularis propria. No lymphadenopathy was identified. Pathology showed a moderately differentiated adenocarcinoma. There was no background BE. The patient was treated on a placebo-controlled, randomized clinical trial of neoadjuvant cisplatin, infusional fluorouracil, and radiotherapy (44 Gy/22 fx) plus investigational study drug, prinomastat (a matrix metalloproteinase inhibitor) or placebo 
for 30 days followed by 4 cycles of weekly paclitaxel and then transhiatal esophagectomy with gastric pull-up and node dissection (Protocol J99-79). The patient was randomized to the placebo. Pathology of the esophagectomy specimen revealed downstaging to stage I, T1N0M0. Twenty-six months after his esophagectomy, the patient was found to have subcarinal lymphadenopathy and bilateral lung nodules on surveillance imaging. He enrolled in a phase II trial of irinotecan and docetaxel days $1 \& 8$, q 21 days (Protocol J00-43). After a brief partial response, and a total of 4 months of treatment, the lung metastases progressed, and his chemotherapy was changed to gemcitabine and docetaxel. He received this regimen for 2 months with further progression. The patient's metastatic lung disease continued to progress and a bronchoscopic biopsy demonstrated lymphangitic spread. He then received capecitabine and oxaliplatin for 2 months with some transient improvement in respiratory symptoms but developed malignant bilateral plural and pericardial effusions that required a pericardial window and repeat thoracenteses and pleurodesis procedures. The disease continued to rapidly progress and the patient expired 1 year from documentation of recurrence. Although not originally tested at the time, we obtained archival biopsy material and performed immunohistochemistry and FISH for ERBB2 as ERBB2 status is now routinely checked in patients with metastatic disease [51]. We found that the patient's primary GEJ tumor was ERBB2 negative by both immunohistochemical staining and FISH amplification (Fig. 1c, d).

\section{GEAMP cells/DNA fingerprinting}

GEAMP cells grew well in monolayer in both media with $5 \%$ serum and in advanced media with $1 \%$ serum. Their typical morphology is shown in Fig. 2. Overall, GEAMP cells had a typical epithelial morphology. Since GEAMP cells were initially serially passaged in mice, we first wanted to confirm that GEAMP cells were similar to the patient's primary tumor. The authenticity of GEAMP cells was determined by comparing the PCR-based STR profiles of GEAMP cells with the archival primary tumor. The original GEJ tumor and GEAMP cells shared $100 \%$ match at 20 loci, confirming the identity of GEAMP cells with its parental tumor (Supplemental Table 1).

\section{Targeted gene sequencing}

Recent genome-wide sequencing projects have shown that EAC, including adenocarcinomas arising in the GEJ, are some of the most highly mutated solid cancers with a high degree of heterogeneity $[16,52,53]$. To understand the mutation profiles of GEAMP cells, we analyzed GEAMP genomic DNA with a UT Southwestern Pan-Cancer assay

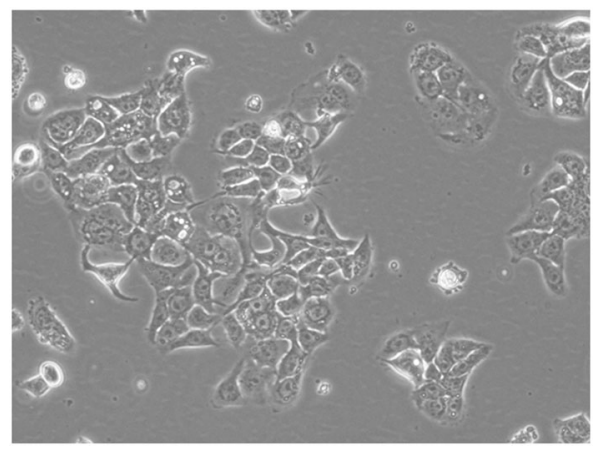

Fig. 2 Light microscopy images of GEAMP cell morphology $(\times 200$ magnification). The cells were grown in Advanced RPMI media with $1 \%$ serum

that covers all exons of 1385 cancer-related genes. All target regions were successfully sequenced. We identified 56 nonsynonymous coding variants in 51 cancer-related genes, including TP53 and APC as previously reported in GEJ cancer [16] (Supplemental Table 2). Copy number variation analysis was also performed using the genomic DNA of GEAMP cells. As shown in Fig. 3a and summarized in Supplemental Table 3, copy number changes were detected at $17 \mathrm{CNV}$ loci including a $49.1-\mathrm{Mb}$ gain encompassing the EGFR gene within chromosome 7 (Fig. 3b). In addition, a 24.7-Mb loss within the chromosome 9p target region was also found in GEAMP cells. This deletion is predicted to cause loss of both alleles of the $C D K N 2 A$ and $C D K N 2 B$ genes. A growing body of work has implicated the tumor suppression function of $C D K N 2 A$ and $C D K N 2 B$ in the pathogenesis of EAC and GEJ cancers [54].

\section{Tumorigencity}

To confirm that GEAMP cells remain tumorigenic, we subcutaneously injected $1 \times 10^{6}$ cells into the flanks of athymic nude and NOD/SCID mice. GEAMP xenografts formed rapidly after injection, being initially seen after 2 weeks. At the time of euthanasia, no obvious macrometastases were found. The histology of H\&E-stained sections of GEAMP flank xenografts were reviewed by two independent pathologists. The staining showed that GEAMP cells formed glandular structures (Fig. 4a, b), similar to the primary tumor. They stained positively with Alcian blue, consistent with mucin production, but did not express CDX2, ERBB2, or TTF1 by immunohistochemistry (Fig. 4c-f). TTF1 negative staining allowed us to exclude the possibility that GEAMP cells originated in an occult lung primary.

Surprisingly, carcinoma cells with squamous differentiation were also observed in the GEAMP xenograft by H\&E staining (Fig. 5a). We next performed 
A
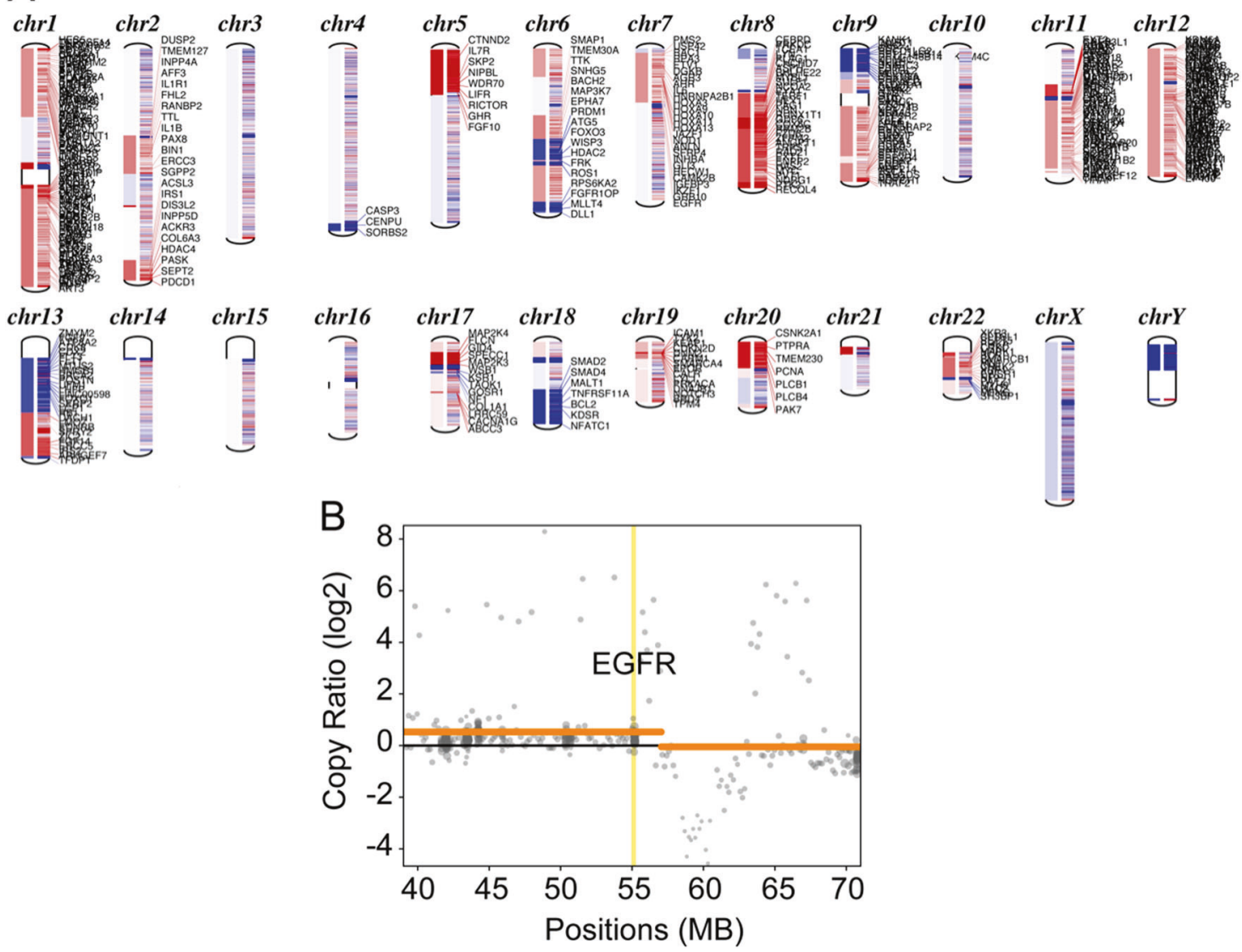

Fig. 3 Analysis of the copy number variations (CNVs) in GEAMP cells. a Subchromosomal deletions (blue) and duplications (red) are observed in the GEAMP genome. b NGS profile for chromosome 7

immunohistochemistry for columnar cell marker CK8/18 and squamous cell marker CK14 on GEAMP flank xenografts. We found that GEAMP xenografts expressed both cytokeratins, but interestingly, CK8/18 positive cells and CK14 positive cells appeared to be mutually exclusive. For example, cells-forming glands were CK8/18 positive and CK14 negative while surrounding cells were CK14 positive and CK8/18 negative in serial sections (Fig. 5b, c). To determine whether GEAMP cells in culture express squamous cytokeratins, we performed western blot analysis of columnar CK8/18, squamous CK13, and squamous CK14 proteins in GEAMP cells as well as in hTERT-immortalized esophageal squamous NES-B10T cells and the esophageal squamous cancer cell line KYSE180. We found columnar marker CK8/18 was more highly expressed in GEAMP cells than in NES-B10T or KYSE180 cells (Fig. 5d). In contrast, squamous suprabasal cell marker CK13 and basal cell marker CK14 were not detected in GEAMP cells (Fig. 5d). Taken together, our results suggest that GEAMP is a GEJ adenocarcinoma cell line but it may contain a subpopulation of cells that have the ability to give rise to both columnar and squamous cells in vivo. Indeed, we disclosing the EGFR gene amplification. Gray points represent individual targeted regions and orange bars represent segments of predicted copy by CNVKit. EGFR gene is highlighted with a yellow line

found that GEAMP xenografts had increased expression of the cancer stem cell (CSC)-associated markers LGR5, CD133, and BMII as compared to the total RNA from whole normal human esophagus (Fig. 5e). When GEAMP cells were grown in vivo, CSCs may have been induced to differentiate into both squamous cell carcinoma and adenocarcinoma.

\section{EGFR inhibitors decrease proliferation of GEAMP Cells}

Although our NGS-based CNV analysis suggested that the EGFR gene was amplified in GEAMP cells (three copies), EGFR was not considered clinically amplified as measured by FISH. Since GEAMP xenografts expressed EGFR by immunohistochemistry (Fig. 6a) and phosphorylated EGFR were readily detected in the GEAMP cell lysate (Fig. 6b), we decided to determine whether treatment of GEAMP cells with the EGFR tyrosine kinase inhibitor, erlotinib, could inhibit the EGFR signaling pathway with functional consequences. GEAMP cells were treated with various concentrations of erlotinib $(0,5,10 \mu \mathrm{M})$ for $48 \mathrm{~h}$. As shown in 
Fig. 4 GEAMP cells can form tumors in a NOD/SCID mouse. Low (a) $\times 100$ and high $(\mathbf{b}) \times 400$ magnification images of H\&Estained section of the GEAMP xenograft. c Alcian blue staining of the GEAMP xenograft $(\times 200)$. Negative immunohistochemical staining of CDX2 (×200) (d), ERBB2 $(\times 400)(\mathbf{e})$, and TTF1 $(\times 200)(\mathbf{f})$, respectively, in the GEAMP xenograft
A

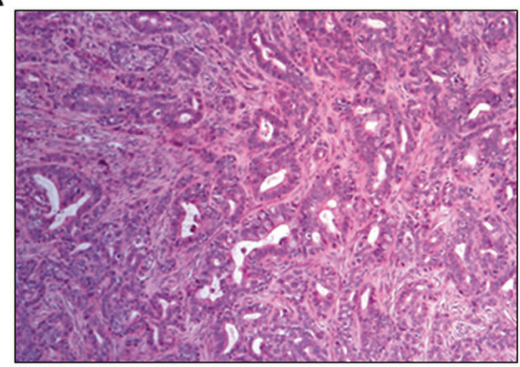

B

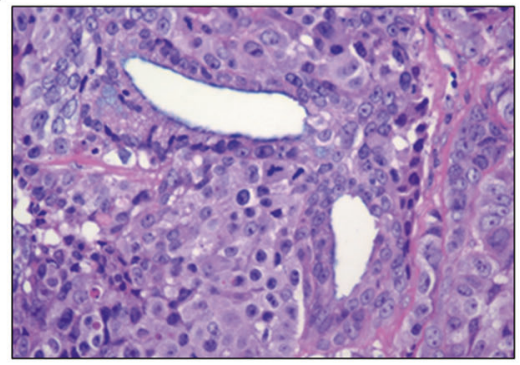

C

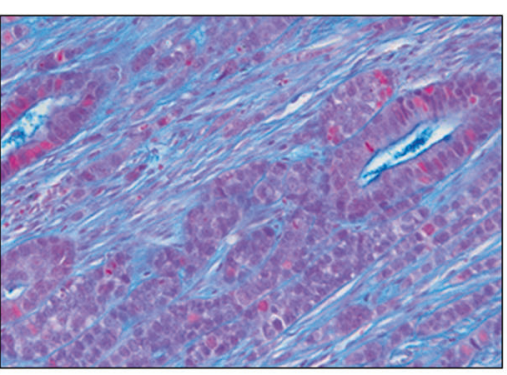

E

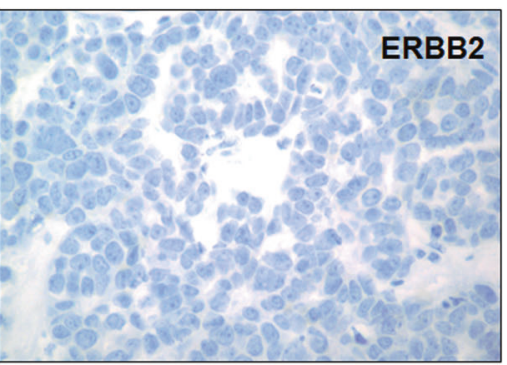

D

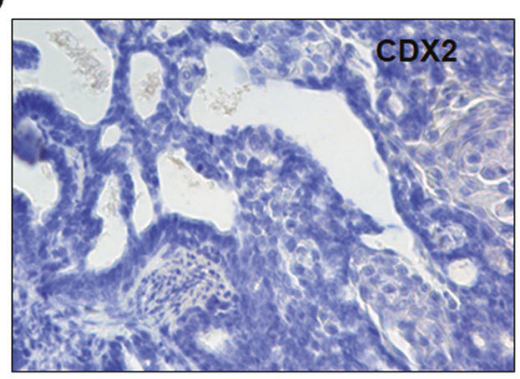

$F$

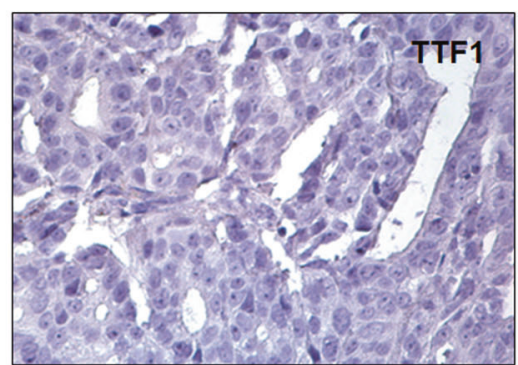

Fig. 6b, erlotinib dramatically inhibited the phosphorylation of EGFR compared with the control. However, the total EGFR protein levels were not changed. To verify whether the inhibition of EGFR phosphorylation was followed by a decrease of EGFR downstream molecules, we next determined the phosphorylation of key components of EGFR signaling including ERK1/2 and AKT in GEAMP cells treated with erlotinib. Surprisingly, erlotinib treatment for $48 \mathrm{~h}$ did not considerably affect the phosphorylation of both ERK1/2 and AKT compared with the control as shown in Fig. 6b. In addition, as shown in Fig. 6d, erlotinib treatment only slightly reduced the cell proliferation of GEAMP cells in vitro compared with the control. Lapatinib is an FDAapproved oral dual tyrosine kinase inhibitor that targets both EGFR and ERBB2. To test whether a different pharmacologic inhibitor of EGFR decreased the oncogenic signaling pathways in GEAMP cells, GEAMP cells were treated with $5 \mu \mathrm{M}$ or $10 \mu \mathrm{M}$ lapatinib for $48 \mathrm{~h}$. As shown in Fig. 6c, lapatinib treatment resulted in a dramatic decrease in the phosphorylation of EGFR, ERK1/2, and AKT. Cell proliferation was also decreased with lapitinib treatment (Fig. 6e). Together, these data suggest that EGFR oncogenic signaling is active in GEAMP cells, and pharmacologic inhibition of this signaling with lapatinib led to reduced cancer cell growth.

\section{Discussion}

Despite the recent advances in therapy for EAC and GEJ cancer, they continue to have a poor prognosis [9, 10]. A major hurdle for understanding the pathogenesis of EAC and GEJ cancer is the lack of available human cell lines and appropriate preclinical animal models. Therefore, the newly described GEAMP cell line is an important reagent for basic and translational EAC and GEJ cancer research. Notably, unlike most of the other established EAC and GEJ cell lines, GEAMP cells were established from a malignant pleural effusion of a GEJ patient and thus, represent metastatic GEJ cancer. This GEJ cancer patient had been heavily treated, making GEAMP a unique cell line derived from tumor cells that have survived multiple doses of radiation and several clinically used chemotherapeutic agents. This is important since the low survival rate of GEJ cancer can be 
Fig. 5 The GEAMP flank xenograft in a NOD/SCID mouse contains both adenocarcinoma and squamous cancer cells. a Representative H\&E-staining image showing the existence of squamous cancer cells in the GEAMP xenograft $(\times 400)$.

Immunohistochemical staining of columnar cell markers CK8/ 18 (b) and squamous cell marker CK14 (c) in the xenograft $(\times 200)$. d Western blot analysis of CK8/18, CK13, and CK14 in NES-B10T, KYSE180, and GEAMP cells. GAPDH is used as a loading control. e qRT-PCR analysis of the cancer stem cell (CSC)-associated genes LGR5, $C D 133$, and BMI1 in GEAMP xenograft and human normal esophagus. Values represent the mean fold change \pm s.e.m. for three experiments relative to GAPDH. Black bars, human normal esophagus; light gray bars, GEAMP xenograft
A
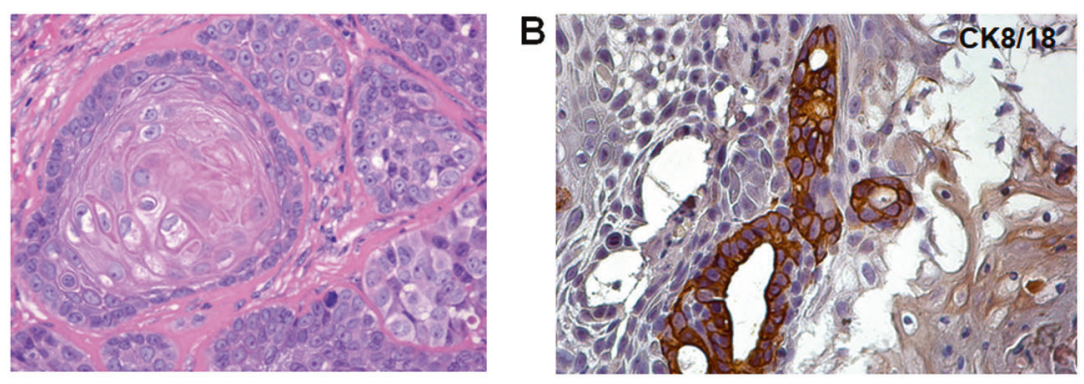

C
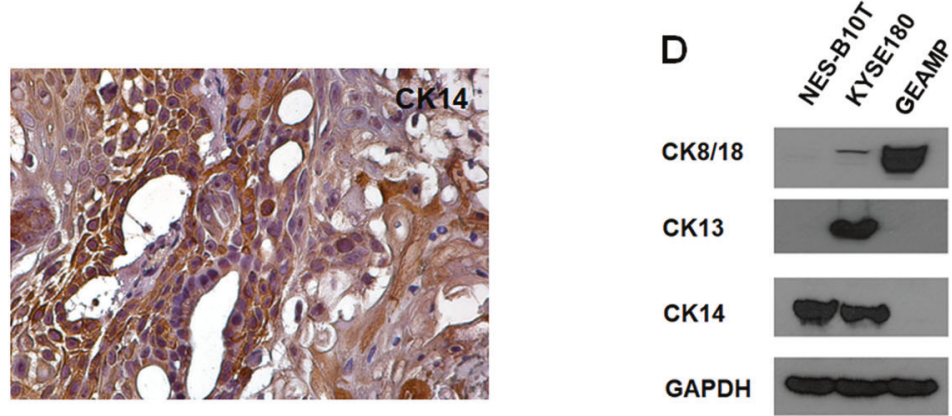

E Xeno vs normal esophagus

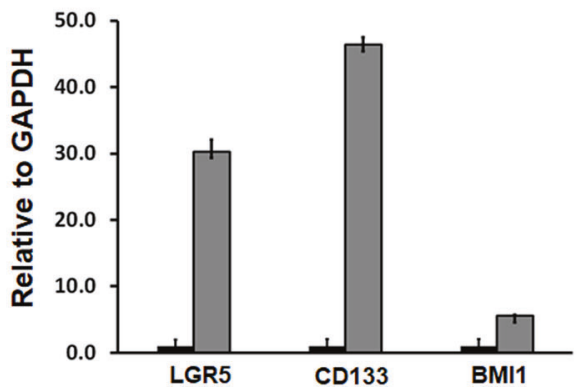

attributed to advanced disease upon initial presentation or acquired chemoresistance in many patients [10, 55]. GEAMP cells were tumorigenic in athymic nude and NODSCID mice when injected subcutaneously. These tumors formed rapidly, within 2 weeks; this is more rapid than other established EAC cell lines such as OE33, FLO-1, and JHEsoAd1 cells. Like many other EAC and GEJ cancer cell lines, GEAMP flank xenografts did not spontaneously metastasize from the site of a subcutaneous injection to other organs in immunodeficient mice.

Knowledge of the mutational landscape of a cell line is critical for designing experiments and correctly interpreting experimental results. Recent genome-wide sequencing projects have demonstrated that EAC and GEJ cancers are some of the most highly mutated solid tumors with a high degree of heterogeneity $[16,52,53]$. To better understand the genetic etiology of GEAMP cells and to assess the presence of therapeutic targets or pathways, we undertook targeted sequencing of the coding regions of 1385 cancerrelated genes. We identified the putative mutations in the exons of these genes by comparison with the human reference genome. Of note, GEAMP cells contained a frameshift mutation in TP53 and a truncating mutation in $A P C$, both of which are frequently altered in GEJ tumors. Therefore, based on these gene alterations, GEAMP cells represent a subgroup of GEJ cancer. Many previous studies have utilized EAC and GEJ cell lines to evaluate the therapeutic efficacy of inhibiting different targets such as EGFR [56], ERBB2 [57], and VEGFR-2 [58]. Here, we utilized GEAMP cells as a preclinical model to assess the effectiveness of EGFR targeted cancer therapy. We successfully inhibited EGFR phosphorylation in GEAMP cells with two FDA-approved EGFR antagonists, erlotinib and lapatinib. The effectiveness of lapatinib on decreasing cell proliferation was also demonstrated in vitro. Based on these experiments, we believe that GEAMP cells will be a useful preclinical tool for evaluating targeted therapy of GEJ cancer.

Remarkably, we found that GEAMP xenografts contained both adenocarcinoma and squamous carcinoma cells determined by H\&E-, CK8/18-, and CK14 staining. In contrast, squamous suprabasal cell marker CK13 and squamous basal cell marker CK14 were not detected in monolayer cultured GEAMP cells. The exact mechanism 
A
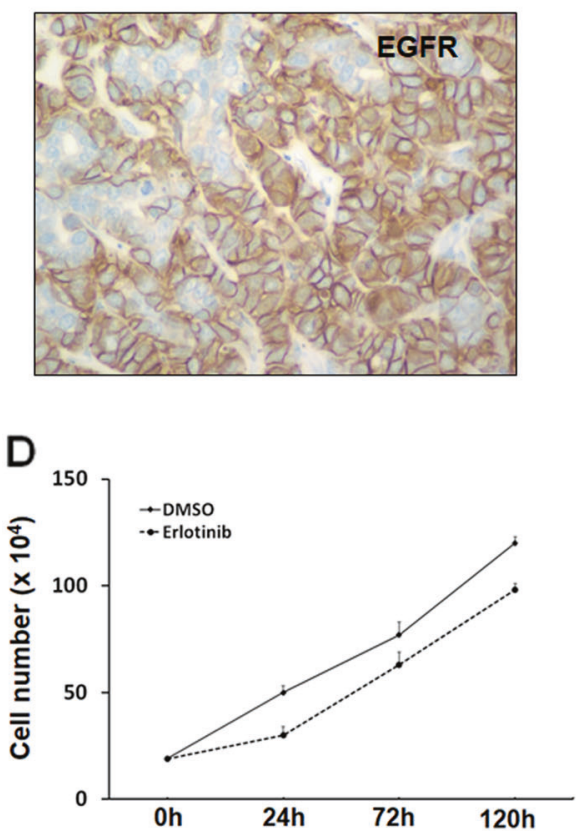

Fig. 6 Tyrosine kinase inhibitors decrease the EGFR signaling pathway and cell proliferation in GEAMP cells. a Immunohistochemical staining of EGFR in the GEAMP xenograft $(\times 400)$. b Western blot analysis of p-EGFR, EGFR, p-ERK1/2, ERK1/2, p-AKT, and AKT protein in GEAMP cells untreated or treated with DMSO, $5 \mu \mathrm{M}$ or $10 \mu \mathrm{M}$ erlotinib for $48 \mathrm{~h}$. c Western blot analysis of p-EGFR, EGFR, p-ERK1/2, ERK1/2, p-AKT, and AKT protein in GEAMP cells

that mediates this phenotype in xenografts remains to be determined, but the CSC hypothesis may help explain this puzzling observation [59, 60]. The general idea of CSC theory is based on clinical and experimental observations, indicating the existence of a subpopulation of cells that can self-renew and differentiate. It suggests that organ-specific stem cells may be the origin of cancer and that cancer develops from maturation disorders of these stem cells. Multiple genetic mouse models suggest that cells near the $\mathrm{SCJ}$ give rise to $\mathrm{BE}$, the precursor of EAC [11]. Whether GEAMP cells originated from stem cells near the SCJ remains unclear. However, the CSC-associated markers LGR5, CD133, and BMI1 were considerably higher in GEAMP xenografts compared with normal human esophagus. Therefore, we hypothesize that at least a subpopulation of GEAMP cells retains the multipotent ability to differentiate into both adenocarcinoma and squamous cancer cells in vivo. These cells may also provide additional insights into the cellular origin of BE, EAC, and GEJ cancer. Alternatively, the patient, from whom GEAMP cells were established had previously received extensive oncologic therapy. Whether this caused the histological transformation from adenocarcinoma to adenosquamous cell carcinoma cannot be excluded. It was previously reported

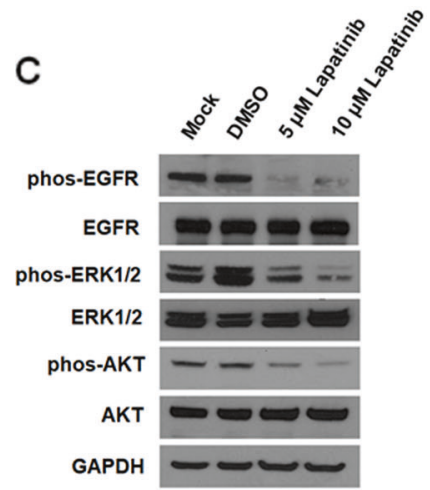

APDH

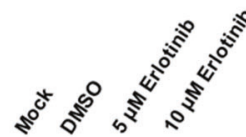

(1)

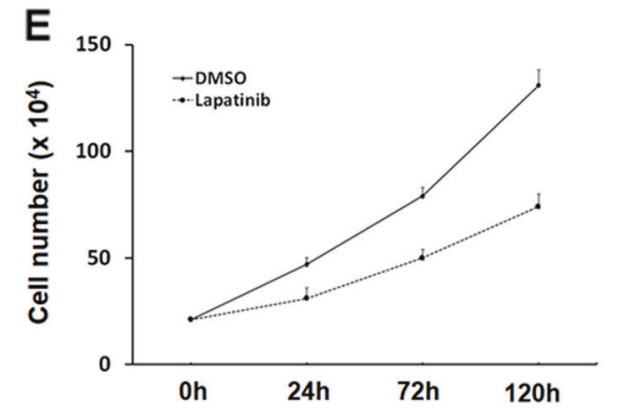

untreated or treated with DMSO, $5 \mu \mathrm{M}$ or $10 \mu \mathrm{M}$ lapatinib for $48 \mathrm{~h}$. GAPDH is used as a loading control. Cell proliferation curve in GEAMP cells treated with $5 \mu \mathrm{M}$ erlotinib (d) or $5 \mu \mathrm{M}$ lapatinib (e) compared to the cells treated with DMSO. Cell counts were carried out on days 1, 3, and 5 after seeding. Data shown are the means \pm SEM of triplicate analyses. Statistical significance was determined by Student's $t$-test. $* P<0.05$

that EGFR mutation-positive lung adenocarcinoma could transform into squamous cell carcinoma following treatment with EGFR tyrosine kinase inhibitors [61]. In addition, a recent study has shown that tumor suppressor $L k b 1$ deficient lung adenocarcinoma cells can progressively transdifferentiate into squamous cancer cells in mouse models [62]. It would be interesting to study whether a similar mechanism happens to GEAMP xenograft cells in the future.

In summary, we have successfully established a GEJ cancer cell line from a malignant pleural effusion from a heavily treated patient with metastatic disease. GEAMP cells can be added to the currently limited pool of EAC and GEJ cancer cell lines and hopefully can be a useful cell line for both basic and translational EAC and GEJ cancer research.

Acknowledgements We thank Beth Cook for assistance in performing Alcian blue staining. We also thank Dr. Rodney Miller and his colleagues at ProPath for performing ERBB2 and EGFR immunohistochemistry and FISH and interpreting the results.

Funding This work was supported by the Career Development Award \#CADE-062-F09 (DHW) from the U.S. Department of Veterans Affairs Biomedical Laboratory Research and Development, the U.S. 
National Institutes of Health R01-DK97340 (DHW), the Simmons Comprehensive Cancer Center and the American Cancer Society ACSIRG-02-196 (DHW), and the Cancer Prevention and Research Institute of Texas (RP150596).

\section{Compliance with ethical standards}

Conflict of interest No relevant conflicts of interest exist. JYP serves as a scientific advisory board member of Miraca Holdings; subsidiaries include Baylor Genetics and Fujirebio, Inc.

Publisher's note: Springer Nature remains neutral with regard to jurisdictional claims in published maps and institutional affiliations.

\section{References}

1. Macdonald JS. Gastric cancer-new therapeutic options. N Engl J Med. 2006;355:76-7.

2. Bray F, Ferlay J, Soerjomataram I, Siegel RL, Torre LA, Jemal A. Global cancer statistics 2018: GLOBOCAN estimates of incidence and mortality worldwide for 36 cancers in 185 countries. CA Cancer J Clin. 2018;68:394-424.

3. Bartel M, Brahmbhatt B, Bhurwal A. Incidence of gastroesophageal junction cancer continues to rise: analysis of surveillance, epidemiology, and end results (SEER) database. J Clin Oncol. 2019;37:40.

4. Sobin LH, Compton CC. TNMseventh edition: what's new, what's changed: communication from the International Union Against Cancer and the American Joint Committee on Cancer. Cancer. 2010;116:5336-9.

5. Ajani JA, D'Amico TA, Almhanna K, Bentrem DJ, Besh S, Chao $\mathrm{J}$, et al. Esophageal and esophagogastric junction cancers, version 1.2015. J Natl Compr Canc Netw. 2015;13:194-227.

6. Rustgi AK, El-Serag HB. Esophageal carcinoma. N Engl J Med. 2014;371:2499-509.

7. Fox MP, van Berkel V. Management of gastroesophageal junction tumors. Surg Clin North Am. 2012;92:1199-212.

8. Spechler SJ, Souza RF. Barrett's esophagus. N Engl J Med. 2014;371:836-45.

9. Lagergren J, Smyth E, Cunningham D, Lagergren P. Oesophageal cancer. Lancet. 2017:390:2383-96.

10. Buas MF, Vaughan TL. Epidemiology and risk factors for gastroesophageal junction tumors: understanding the rising incidence of this disease. Semin Radiat Oncol. 2013;23:3-9.

11. Zhang W, Wang DH. Origins of metaplasia in Barrett's esophagus: is this an esophageal stem or progenitor cell disease? Dig Dis Sci. 2018;63:2005-12.

12. Huang Q, Fan X, Agoston AT, Feng A, Yu H, Lauwers G, et al. Comparison of gastro-oesophageal junction carcinomas in Chinese versus American patients. Histopathology. 2011;59:188-97.

13. Gavin AT, Francisci S, Foschi R, Donnelly DW, Lemmens V, Brenner $\mathrm{H}$, et al. Oesophageal cancer survival in Europe: a EUROCARE-4 study. Cancer Epidemiol. 2012;36:505-12.

14. Njei B, McCarty TR, Birk JW. Trends in esophageal cancer survival in United States adults from 1973 to 2009: a SEER database analysis. J Gastroenterol Hepatol. 2016;31:1141-6.

15. Siegel RL, Miller KD, Jemal A. Cancer statistics, 2019. CA Cancer J Clin. 2019;69:7-34.

16. Cancer Genome Atlas Research N, Analysis Working Group: Asan U, Agency BCC, Brigham, Women's H, Broad I, et al. Integrated genomic characterization of oesophageal carcinoma. Nature. 2017;541:169-75.
17. Stachler MD, Taylor-Weiner A, Peng S, McKenna A, Agoston AT, Odze RD, et al. Paired exome analysis of Barrett's esophagus and adenocarcinoma. Nat Genet. 2015;47:1047-55.

18. Wang K, Johnson A, Ali SM, Klempner SJ, Bekaii-Saab T, Vacirca JL, et al. Comprehensive genomic profiling of advanced esophageal squamous cell carcinomas and esophageal adenocarcinomas reveals similarities and differences. Oncologist. 2015;20:1132-9.

19. Li-Chang HH, Kasaian K, Ng Y, Lum A, Kong E, Lim H, et al. Retrospective review using targeted deep sequencing reveals mutational differences between gastroesophageal junction and gastric carcinomas. BMC Cancer. 2015;15:32.

20. Quante M, Bhagat G, Abrams JA, Marache F, Good P, Lee MD, et al. Bile acid and inflammation activate gastric cardia stem cells in a mouse model of Barrett-like metaplasia. Cancer Cell. 2012;21:36-51.

21. Wang X, Ouyang H, Yamamoto Y, Kumar PA, Wei TS, Dagher $\mathrm{R}$, et al. Residual embryonic cells as precursors of a Barrett's-like metaplasia. Cell. 2011;145:1023-35.

22. Jiang M, Li H, Zhang Y, Yang Y, Lu R, Liu K, et al. Transitional basal cells at the squamous-columnar junction generate Barrett's oesophagus. Nature. 2017;550:529-33.

23. Boonstra JJ, van Marion R, Beer DG, Lin L, Chaves P, Ribeiro C, et al. Verification and unmasking of widely used human esophageal adenocarcinoma cell lines. $J$ Natl Cancer Inst. 2010;102:271-4.

24. Hughes SJ, Nambu Y, Soldes OS, Hamstra D, Rehemtulla A, Iannettoni MD, et al. Fas/APO-1 (CD95) is not translocated to the cell membrane in esophageal adenocarcinoma. Cancer Res. 1997;57:5571-8.

25. Shimada $Y$, Imamura $M$, Wagata $T$, Yamaguchi $N$, Tobe $T$. Characterization of 21 newly established esophageal cancer cell lines. Cancer. 1992;69:277-84.

26. Altorki N, Schwartz GK, Blundell M, Davis BM, Kelsen DP, Albino AP. Characterization of cell lines established from human gastric-esophageal adenocarcinomas. Biologic phenotype and invasion potential. Cancer. 1993;72:649-57.

27. Rockett JC, Larkin K, Darnton SJ, Morris AG, Matthews HR. Five newly established oesophageal carcinoma cell lines: phenotypic and immunological characterization. $\mathrm{Br} \mathrm{J}$ Cancer. 1997;75:258-63.

28. Alvarez H, Koorstra JB, Hong SM, Boonstra JJ, Dinjens WN, Foratiere AA, et al. Establishment and characterization of a bona fide Barrett esophagus-associated adenocarcinoma cell line. Cancer Biol Ther. 2008;7:1753-5.

29. de Both NJ, Wijnhoven BP, Sleddens HF, Tilanus HW, Dinjens WN. Establishment of cell lines from adenocarcinomas of the esophagus and gastric cardia growing in vivo and in vitro. Virchows Arch. 2001;438:451-6.

30. Wijnhoven BP, Tilanus MG, Morris AG, Darnton SJ, Tilanus HW, Dinjens WN. Human oesophageal adenocarcinoma cell lines JROECL 47 and JROECL 50 are admixtures of the human colon carcinoma cell line HCT 116. Br J Cancer. 2000;82:1510-2.

31. Clemons NJ, Do H, Fennell C, Deb S, Fellowes A, Dobrovic A, et al. Characterization of a novel tumorigenic esophageal adenocarcinoma cell line: OANC1. Dig Dis Sci. 2014;59:78-88.

32. Garcia E, Hayden A, Birts C, Britton E, Cowie A, Pickard K, et al. Authentication and characterisation of a new oesophageal adenocarcinoma cell line: MFD-1. Sci Rep. 2016;6:32417.

33. Liu DS, Duong CP, Phillips WA, Clemons NJ. Preclinical models of esophageal adenocarcinoma for drug development. Discov Med. 2016;22:371-9.

34. Li H. Aligning sequence reads, clone sequences and assembly contigs with BWA-MEM. arXiv. 2013;3:13033997. 
35. Li H, Handsaker B, Wysoker A, Fennell T, Ruan J, Homer N, et al. The sequence alignment/map format and SAMtools. Bioinformatics. 2009;25:2078-9.

36. DePristo MA, Banks E, Poplin R, Garimella KV, Maguire JR, Hartl $\mathrm{C}$, et al. A framework for variation discovery and genotyping using next-generation DNA sequencing data. Nat Genet. 2011;43:491-8.

37. McKenna A, Hanna M, Banks E, Sivachenko A, Cibulskis K, Kernytsky A, et al. The genome analysis toolkit: a MapReduce framework for analyzing next-generation DNA sequencing data. Genome Res. 2010;20:1297-303.

38. Rimmer A, Phan H, Mathieson I, Iqbal Z, Twigg SRF, Consortium WGS, et al. Integrating mapping-, assembly- and haplotype-based approaches for calling variants in clinical sequencing applications. Nat Genet. 2014;46:912-8.

39. Chiang C, Layer RM, Faust GG, Lindberg MR, Rose DB, Garrison $\mathrm{EP}$, et al. SpeedSeq: ultra-fast personal genome analysis and interpretation. Nat Methods. 2015;12:966-8.

40. Saunders CT, Wong WS, Swamy S, Becq J, Murray LJ, Cheetham RK. Strelka: accurate somatic small-variant calling from sequenced tumor-normal sample pairs. Bioinformatics. 2012;28:1811-7.

41. Cibulskis K, Lawrence MS, Carter SL, Sivachenko A, Jaffe D, Sougnez C, et al. Sensitive detection of somatic point mutations in impure and heterogeneous cancer samples. Nat Biotechnol. 2013;31:213-9.

42. Hansen NF, Gartner JJ, Mei L, Samuels Y, Mullikin JC. Shimmer: detection of genetic alterations in tumors using next-generation sequence data. Bioinformatics. 2013;29:1498-503.

43. Reble E, Castellani CA, Melka MG, O'Reilly R, Singh SM. VarScan2 analysis of de novo variants in monozygotic twins discordant for schizophrenia. Psychiatr Genet. 2017;27:62-70.

44. Kim S, Jeong K, Bhutani K, Lee J, Patel A, Scott E, et al. Virmid: accurate detection of somatic mutations with sample impurity inference. Genome Biol. 2013;14:R90.

45. Cingolani P, Platts A, Wang le L, Coon M, Nguyen T, Wang L, et al. A program for annotating and predicting the effects of single nucleotide polymorphisms, SnpEff: SNPs in the genome of Drosophila melanogaster strainw1118; iso-2; iso-3. Fly. 2012;6: 80-92.

46. Haas B, Dobin A, Stransky N, Li B, Yang X, Tickle T, et al. STARfusion: fast and accurate fusion transcript detection from RNA-seq. https://www.biorxiv.org/content/10.1101/120295v1. 2017.

47. Talevich E, Shain AH, Botton T, Bastian BC. CNVkit: genomewide copy number detection and visualization from targeted DNA sequencing. PLoS Comput Biol. 2016;12:e1004873.

48. Cheng DT, Prasad M, Chekaluk Y, Benayed R, Sadowska J, Zehir A, et al. Comprehensive detection of germline variants by MSKIMPACT, a clinical diagnostic platform for solid tumor molecular oncology and concurrent cancer predisposition testing. BMC Med Genomics. 2017;10:33.
49. Lek M, Karczewski KJ, Minikel EV, Samocha KE, Banks E, Fennell T, et al. Analysis of protein-coding genetic variation in 60,706 humans. Nature. 2016;536:285-91.

50. Zhang W, Zeng X, Briggs KJ, Beaty R, Simons B, Chiu Yen RW, et al. A potential tumor suppressor role for Hicl in breast cancer through transcriptional repression of ephrin-A1. Oncogene. 2010;29:2467-76.

51. Bang YJ, Van Cutsem E, Feyereislova A, Chung HC, Shen L, Sawaki A, et al. Trastuzumab in combination with chemotherapy versus chemotherapy alone for treatment of HER2-positive advanced gastric or gastro-oesophageal junction cancer (ToGA): a phase 3, open-label, randomised controlled trial. Lancet. 2010;376:687-97.

52. Dulak AM, Stojanov P, Peng S, Lawrence MS, Fox C, Stewart C, et al. Exome and whole-genome sequencing of esophageal adenocarcinoma identifies recurrent driver events and mutational complexity. Nat Genet. 2013;45:478-86.

53. Weaver JMJ, Ross-Innes CS, Shannon N, Lynch AG, Forshew T, Barbera $\mathrm{M}$, et al. Ordering of mutations in preinvasive disease stages of esophageal carcinogenesis. Nat Genet. 2014;46:837-43.

54. Suzuki H, Zhou X, Yin J, Lei J, Jiang HY, Suzuki Y, et al. Intragenic mutations of $\mathrm{CDKN} 2 \mathrm{~B}$ and $\mathrm{CDKN} 2 \mathrm{~A}$ in primary human esophageal cancers. Hum Mol Genet. 1995;4:1883-7.

55. Ustaalioglu BBO, Tilki M, Surmelioglu A, Bilici A, Gonen C, Ustaalioglu $\mathrm{R}$, et al. The clinicopathologic characteristics and prognostic factors of gastroesophageal junction tumors according to Siewert classification. Turk J Surg. 2017;33:18-24.

56. Ekman S, Bergqvist M, Heldin CH, Lennartsson J. Activation of growth factor receptors in esophageal cancer-implications for therapy. Oncologist. 2007;12:1165-77.

57. Gros SJ, Kurschat N, Dohrmann T, Reichelt U, Dancau AM, Peldschus K, et al. Effective therapeutic targeting of the overexpressed HER-2 receptor in a highly metastatic orthotopic model of esophageal carcinoma. Mol Cancer Ther. 2010;9:2037-45.

58. Janjigian YY, Vakiani E, Ku GY, Herrera JM, Tang LH, Bouvier $\mathrm{N}$, et al. Phase II trial of sorafenib in patients with chemotherapy refractory metastatic esophageal and gastroesophageal (GE) junction cancer. PLoS ONE. 2015;10:e0134731.

59. Qian X, Tan C, Wang F, Yang B, Ge Y, Guan Z, et al. Esophageal cancer stem cells and implications for future therapeutics. Onco Targets Ther. 2016;9:2247-54.

60. Harada K, Pool Pizzi M, Baba H, Shanbhag ND, Song S, Ajani JA. Cancer stem cells in esophageal cancer and response to therapy. Cancer. 2018;124:3962-4.

61. Haratani K, Hayashi H, Watanabe S, Kaneda H, Yoshida T, Takeda M, et al. Two cases of EGFR mutation-positive lung adenocarcinoma that transformed into squamous cell carcinoma: successful treatment of one case with rociletinib. Ann Oncol. 2016;27:200-2.

62. Han X, Li F, Fang Z, Gao Y, Li F, Fang R, et al. Transdifferentiation of lung adenocarcinoma in mice with Lkb1 deficiency to squamous cell carcinoma. Nat Commun. 2014;5:3261. 